\title{
Una mirada al examen profesional por áreas de conocimiento en la licenciatura en Enfermería
}

\author{
A look to the professional Exam through \\ its knowledge areas
}

Mtra. Ma Antonia González Noriega*, Lic. Yazmin Nicolás Caballero** y Lic. Octavio Rodríguez Arellano**

* Técnico Académico Asociado C, TC. y ** Técnicos Académicos Asociados A, TC. Integrantes de la Unidad de Apoyo para la Evaluación de los Aprendizajes.

\section{Resumen}

El Examen General por Áreas de Conocimiento (EPAC) es una opción de titulación que ofrece la Escuela Nacional de Enfermería y Obstetricia de la UNAM a estudiantes de los sistemas escolarizado, abierto e incorporado, para obtener el grado de licenciatura o profesional técnico en Enfermería. El propósito del artículo es socializar y evidenciar el trabajo realizado con el EPAC de nivel licenciatura a partir de la creación de la Unidad de Apoyo para la Evaluación de los Aprendizajes. Ello ha permitido, con el trabajo de un equipo multidisciplinar, mejorar el proceso del examen, hacer innovaciones informáticas y administrativas, mantener un apoyo educativo directo y confiable tanto a instituciones participantes como a sustentantes.

The General Test by Knowledge Areas (EPAC) is an option for obtaining the nurse licentiate or technician degree that the National Nursery and Obstetrics School (ENEO, UNAM) offers to the students of the traditional, open and incorporated syste$m s$. The purpose of this article is to share what has been done regarding the licentiate level EPAC since the Support Unit for Knowledge Evaluation was created. This has allowed, with the work of a multidisciplinary team, to improve the general exam process, to attain computer and administrative innovations and to give a direct, realiable educative support to both institutions and individual participants.
Los resultados que se muestran fueron extraídos de las cuatro aplicaciones realizadas hasta el año 2006, las que se juzgaron a partir de estudios de tipo descriptivo y transversal, con muestra censal.

Es innegable que el trabajo permanente con este proceso reorientará las acciones para una mejora continua, que posibiliten altos estándares de confiabilidad lo que garantizará la obtención de resultados más objetivos y oportunos.

Palabras clave: Examen Profesional por Áreas de Conocimiento, reactivos, áreas de conocimiento, calidad en la formación de profesionales en Enfermería.

\section{Abstract}

The results shown in this article proceed from the four exams presented until the year 2006, which were judged based on descriptive, transversal studies with census sample.

It is undeniable that the permanent work with this process will redirect the actions for a continuous improvement, that will allow high reliability standards that guarantee the attainment of more objective, convenient results.

Key words: General Test by Knowledge Areas, test item, knowledge areas, quality in the education of nursery professionals. 


\section{INTRODUCCIÓN}

Una de las tendencias educativas en el ámbito de la Educación Superior está dirigida a la apertura de las escuelas y facultades para ofrecer a su población estudiantil nuevos procesos viables como opciones de titulación de las diferentes carreras, debido a que hay un mayor crecimiento de la matrícula de pregrado y al problema que constituye el creciente porcentaje de alumnos que no logran obtener el grado de licenciatura, quedándose como pasantes. Con el afán de revertir esta tendencia se han propuesto diversas opciones de titulación como: elaboración de tesis, tesina, informes de actividades profesionales, informe de servicio social, por totalidad de créditos y alto nivel académico, mediante estudios de posgrado, y mediante la realización de exámenes generales de conocimientos ${ }^{\mathrm{a}}$.

La Escuela Nacional de Enfermería y Obstetricia (ENEO) ha ofrecido como vía de titulación el Examen General por Áreas de Conocimiento (EPAC). Desde su implementación, se ha constatado una demanda constante y permanente por lo que ha sido necesario seguir renovando las formas de trabajo que garanticen una mayor confiabilidad en el instrumento y los resultados. El presente artículo describe el trabajo y análisis realizado por la Unidad de Apoyo para la Evaluación de los Aprendizajes con respecto al EPAC.

\section{Un poco de historia}

Desde 1987 se ha venido aplicando el EPAC de forma regular, apoyados en un principio por la Facultad de Medicina de la UNAM y por el CENEVAL. Más adelante, en 1998, con la compra del lector óptico para la lectura de las Hojas de Respuesta y del programa Kalt para calificación de alumnos y reactivos, todo el proceso comenzó a realizarse en la ENEO, involucrando a diferentes unidades. Por último se crea en el año 2005 la Unidad de Apoyo para la Evaluación de los Aprendizajes, encargada de la organización, aplicación, emisión y análisis de resultados, para tener un mayor control en todo el proceso.

Un primer paso para construir un instrumento de evaluación válido y confiable, fue la creación de un software que permitiera calificar los exámenes y calibrar los reactivos y tener así la información necesaria para su posterior análisis. Contando con el software, se leyeron los exámenes de la aplicación de noviembre de 2004, cuyos resultados sirvieron como base para conformar el examen previsto para junio de 2005, el cual se integró con $30 \%$ de reactivos bien calificados $^{\text {b }}$. Posteriormente se estableció un padrón de distractores, tablas de equivalencia y se generó una encuesta que se incorporó al examen que se utilizó para obtener información de los sustentantes que permitiera mejorar el proceso.

Con un trabajo de organización y sistematización se logró incrementar la base de reactivos aceptados, tomando en cuenta su grado de dificultad y poder de discriminación. Así fueron empleados en el EPAC de junio de 2005 el 30\% de reactivos considerados como buenos, en diciembre de 2005 el 43\%; junio 2006 el 38\% y diciembre 2006 el 51\%. El resto de los reactivos fueron proporcionados por la planta docente de la ENEO o reactivos de otras aplicaciones que fueron modificados. Esta situación ha permitido garantizar cada vez mayor confiabilidad y seguridad del instrumento y los resultados obtenidos.

Cabe señalar que en la aplicación de junio de 2005 el examen estuvo conformado por cuatro partes: Enfermería Fundamental, Enfermería del adolescente, adulto y anciano, Enfermería materno-infantil y por las de apoyo a la Enfermería, abarcando todas las asignaturas obligatorias señaladas en el Plan de Estudio ${ }^{\circ}$.

Sin embargo debido a que esta división ocasionaba diferentes problemas, se redujeron las secciones a dos, a partir de la aplicación de diciembre de 2005. Esta decisión se fundamentó en dos supuestos: a) reducir los niveles de estrés presentados por los sustentantes al resolver cuatro diferentes cuadernillos, y b) reducir la posibilidad de error al llenar sólo dos Hojas de Respuesta en lugar de cuatro, con lo que se conseguiría disminuir la omisión o inexactitud de un dato, lo

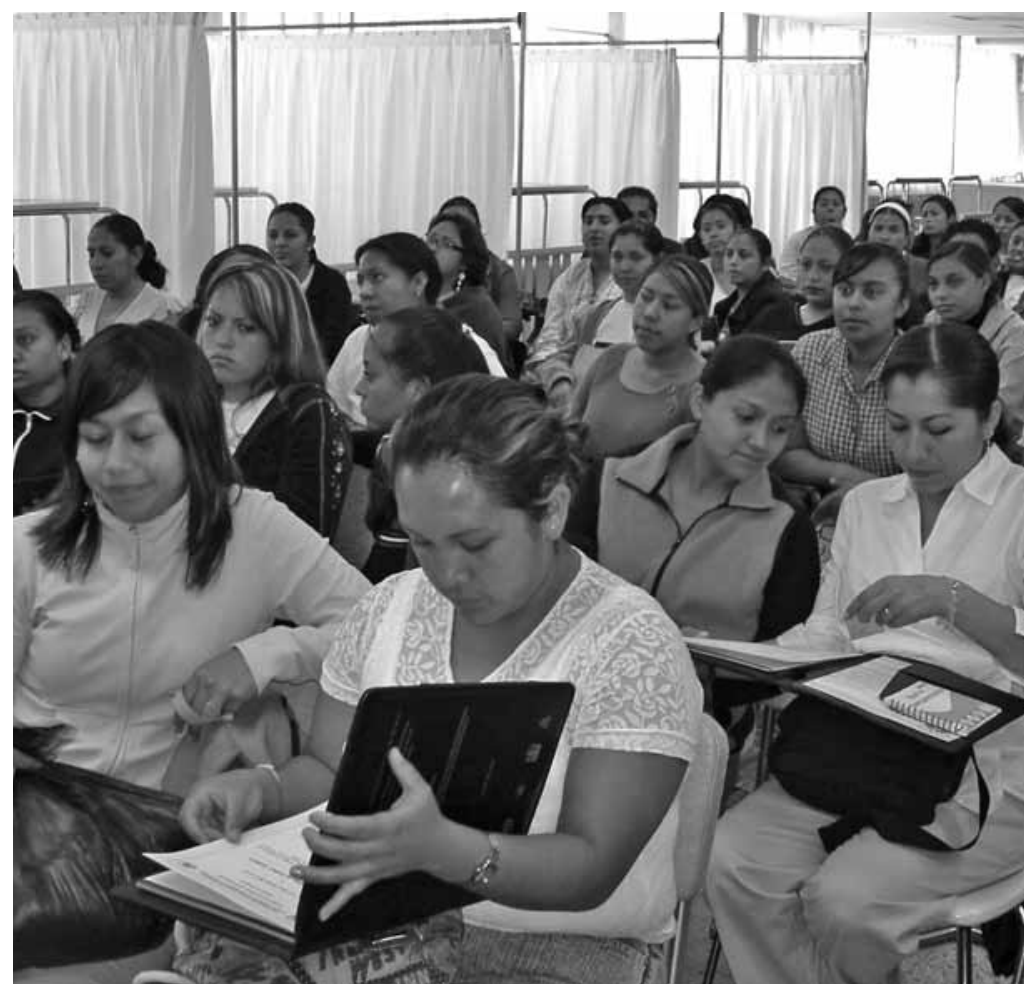


que en anteriores aplicaciones constituía un obstáculo para la aprobación del examen, ya que se invalidaba esa hoja de respuesta, además que aportaba datos erróneos para poder calificar los reactivos. Hubo que rediseñar la Hoja de Respuestas, simplificando el llenado de los datos generales y agrupando las asignaturas de una forma más lógica y fácil de calificar por áreas de conocimiento, aunque se mantuvo el mismo número de reactivos. Los seis conjuntos ${ }^{d}$ en los que se agruparon los reactivos fueron:

- Fundamentos de Enfermería

- Enfermería materno-infantil

- Enfermería del adolescente, adulto y anciano (AAA)

- Ciencias biológicas

- Formación instrumental

- Formación humanística

\section{Los reactivos}

El EPAC es un examen objetivo conformado por reactivos de opción múltiple, mismos que son formulados y seleccionados en distintos grados de complejidad para medir conocimientos y habilidades que se consideran indispensables para garantizar el ejercicio de Enfermería.

El examen está conformado por dos secciones con dos versiones cada una y concentran un total de 280 reactivos, los cuales se distribuyen conforme al número de créditos que tiene cada asignatura. Como ejemplo: Obstetricia I, con 36 créditos, $9.30 \%$ de la totalidad de los establecidos, le corresponde 22 reactivos distribuidos en 17 preguntas independientes y 5 sujetos a un estudio de caso. Además cuenta con ocho estudios de caso para explorar principalmente el Proceso de Atención de Enfermería.

\section{MÉTODO}

Se realizó un estudio de tipo descriptivo y transversal, con muestra censal. La técnica de recolección de datos se efectuó con base en análisis documental y registro sistemático. Los instrumentos empleados fueron la tabla de concentración de datos numéricos de los resultados obtenidos por el lector óptico y el software, y del historial académico de cada sustentante. Los datos se sometieron a un tratamiento analítico que responden a los procesos de calificación, registro y codificación de datos y el análisis estadístico se realizó a través de la curva normal, con un margen de error de 0.05 .

EI EPAC se mide con una escala que va de 300 a 775 puntos, siendo el punto donde se determina la relación
Aprobados-No aprobados, 420. A partir de la puntuación 700 o más, se considera de excelencia.

El modelo de evaluación está sustentado dentro de la evaluación del producto que es una medición, interpretación y juicio acerca del cumplimiento de los objetivos y de la eficacia de la enseñanza para la toma de decisionese5,6. La evaluación fue sumativa al ser realizada después de un periodo de aprendizaje en la finalización de la carrera.

\section{RESULTADOS}

Desde la creación de la unidad se han aplicado cuatro EPAC $^{47}$ con la participación de 2,716 sustentantes, pertenecientes a los sistemas escolarizado, abierto e incorporado (Cuadro 1)

El examen se ha realizado en diferentes sedes de la República, a las que acuden profesores pertenecientes a la ENEO que fungen como aplicadores. Muchas veces se concentran dos o más sedes en un solo punto, como en diciembre de 2006 en que la ENEO agrupó 14 diferentes instituciones y sistemas; o en junio de 2006 que de 56 sedes, se agruparon 34. (Cuadro 2)

CUADRO 1.

Número de sustentantes por sistema y aplicación.

\begin{tabular}{|l|c|c|c|c|l|}
\hline Aplicación & $\begin{array}{l}\text { J u n } \\
\mathbf{2 0 0 5}\end{array}$ & $\begin{array}{l}\text { D i c } \\
\mathbf{2 0 0 5}\end{array}$ & $\begin{array}{l}\text { J u n } \\
\mathbf{2 0 0 6}\end{array}$ & $\begin{array}{l}\text { D i c } \\
\mathbf{2 0 0 6}\end{array}$ & TOTAL \\
\hline Escolarizado & 3 & 102 & 69 & 88 & 262 \\
\hline Abierto & 1 & 341 & 343 & 88 & 773 \\
\hline Incorporado & 566 & 0 & 992 & 123 & 1,681 \\
\hline TOTALES & 570 & 443 & 1404 & 299 & 2,716 \\
\hline
\end{tabular}

Fuente: resultados obtenidos de las Hojas de respuesta.

CUADRO 2.

Número de sedes en cada aplicación

\begin{tabular}{|l|c|}
\hline \multicolumn{1}{|c|}{ Aplicación } & Número de sedes \\
\hline Junio 2005: & 19 \\
\hline Diciembre 2005: & 6 \\
\hline Junio 2006: & 56 \\
\hline Diciembre 2006: & 3 \\
\hline
\end{tabular}

Fuente: registro de aspirantes del archivo de la Unidad de Apoyo para la Evaluación de los aprendizajes. 
El área de conocimientos en donde se obtienen mejores resultados es Enfermería del adolescente, adulto y anciano, y en donde se muestra el desempeño más bajo es Ciencias biológicas. (Gráfica 1)

\section{Gráfica 1.}

Puntajes obtenidos por áreas de conocimiento en los distintos sistemas de los últimos tres EPAC. También se muestra el número de sustentantes.

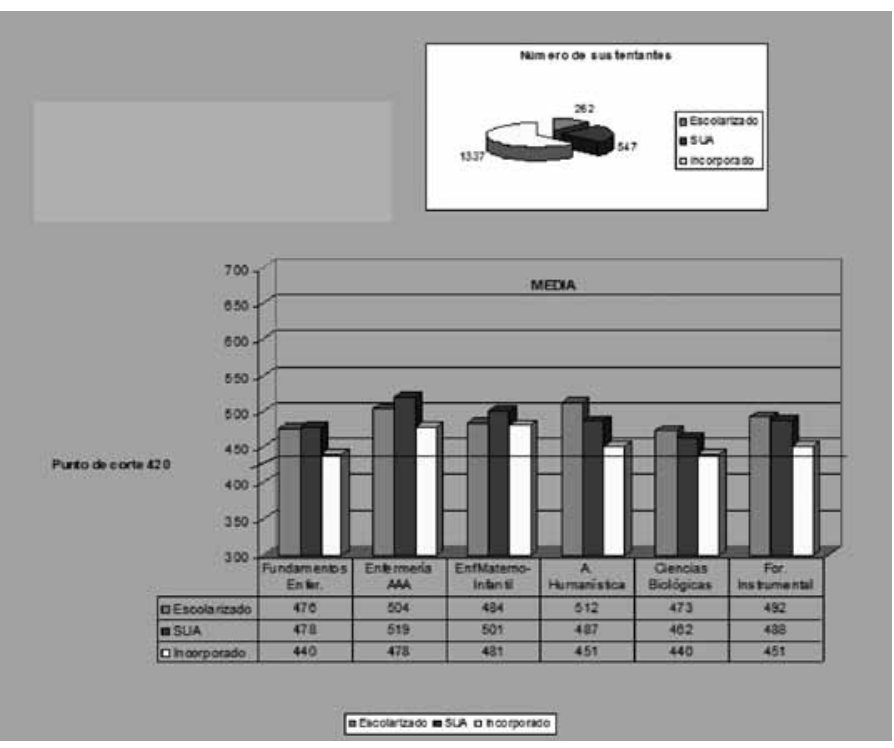

Del total de la población, $77.91 \%$ han aprobado, de ella el $0.075 \%$ ha alcanzado la puntuación de excelenciag8 ${ }^{98}$

Con base en el rastreo de los promedios de carrera de los sustentantes en comparación con las puntuaciones alcanzadas en el examen, se realiza un análisis en el que se manifiesta un contraste entre estos dos resultados: el mayor número de aprobados se concentra en un promedio de carrera que va de 7.1 a 8.5 , siendo que la parte alta de los promedios de carrera (8.6 a 10) concentra a menos de un $4 \%$ de la población. (Gráfica 2)

\section{GRÁFICA 2.}

Comparativo de porcentaje de sustentantes entre promedios de carrera por cada examen aplicado y puntajes obtenidos en el EPAC.

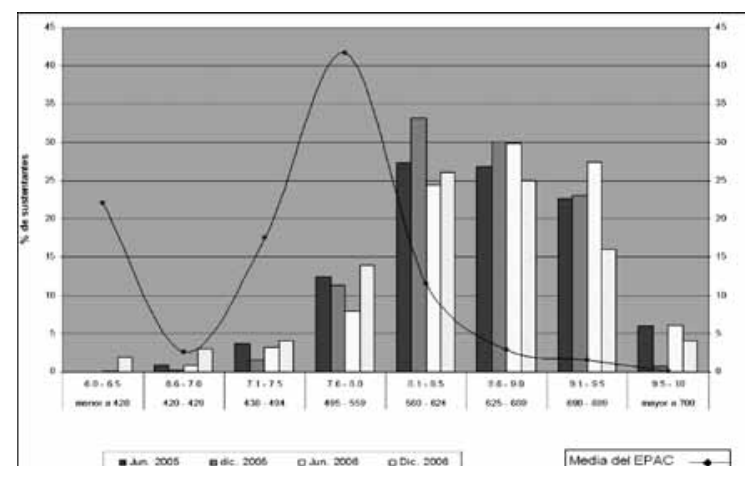

\section{Innovaciones}

Debido al crecimiento de la demanda de los pasantes que ven en el examen una opción viable para su titulación y con el afán de ofrecerles un mejor servicio, se han realizado algunas acciones oportunas y viables que han contribuido al mejoramiento tanto del proceso del examen como al fortalecimiento de la formación de los estudiantes de Enfermería:

a) Informes estadísticos a las instituciones.

Con la finalidad de contribuir a la calidad en la formación de los profesionales de Enfermería, se ha puesto a disposición de autoridades de sistemas y sedes que participaron en determinada aplicación del examen, los resultados generales que obtuvieron. Para ello, la información se ha agrupado en las seis áreas de conocimiento que conforman el EPAC, mostrando en cada una de ellas el puntaje promedio que obtuvo su población y una gráfica de quintiles para poder observar el desplazamiento de la curva comparada con la curva normal (Ejemplo gráfica 3). La intención esencial de este ejercicio es que los datos sirvan de referencia particular para que se puedan reforzar aquellos aprendizajes y/o temáticas que con sustento estadístico, muestran bajas puntuaciones.

b) Resultados en línea.

Desde la aplicación de diciembre de 2005 se da a conocer, después de 15 días de haber presentado el examen, el resultado obtenido por cada sustentante a través de la página de la ENEO ${ }^{\text {h9 }}$. De esta forma pronta y confidencial, cada sustentante puede utilizar esa información del modo que más le convenga.

c) Informe a sustentantes No aprobados.

Con la intención de brindar un apoyo más directo a los sustentantes que no aprobaron el examen y que deseen presentarlo en aplicaciones posteriores, se les proporciona un informe en el que se detallan las puntuaciones obtenidas en cada área de conocimiento, especificando las asignaturas que la conforman. El objetivo es que el sustentante conozca en qué área necesita mayor preparación pudiendo así incrementar la posibilidad de aprobarlo en la siguiente oportunidad.

\section{d) Correo electrónico para orientación.}

Se creó la cuenta de correo electrónico epac@eneo. unam. $m x$ para brindar a los sustentantes un espacio en el que puedan escribir dudas, comentarios y orientacio- 
nes varias, antes y después de la aplicación del examen. Con este medio se garantiza una respuesta oportuna lo cual ha permitido aminorar las fallas, obstáculos y/o errores propios de procesos de esta dimensión.

\section{e) Guía en línea.}

A partir de la aplicación de junio de 2006, se incorporó a la página de la ENEO la Guía de Estudio oficial para presentar el EPAC con la intención de que esté a disposición de los sustentantes con suficiente anticipación que les permita prepararse para presentar el examen.

\section{COMENTARIO FINAL}

Para los integrantes de la Unidad de Apoyo para la Evaluación de los Aprendizajes es claro que para llevar a cabo cada una de las aplicaciones del EPAC se debe realizar con todo el compromiso y formalidad, ya que se construye un instrumento evaluativo de alto impacto, porque con él se determina si una persona es "apta" para recibir el título de licenciada(o) o de profesional técnico en Enfermería, lo que evidentemente tendrá un efecto directo tanto en su vida personal como en el ejercicio profesional.

Por tal motivo, se ha buscado un trabajo conjunto con los diferentes sistemas que participan en el examen: escolarizado, abierto e incorporado a través de la Dirección General de Incorporación y Revalidación de Estudios (DGlRE-UNAM), para garantizar procesos exitosos en cuanto a logística, organización, aplicación y emisión de resultados.

Así mismo, se han enfocado los esfuerzos para construir un instrumento adecuado, que se sustente en una base significativa de reactivos bien calificados y que dé plena seguridad de que los resultados que se obtengan son confiables y oportunos.

\section{GRÁFICA 3.}

Ejemplo de una sede que muestra la distribución de su población sustentante del EPAC con respecto a la curva normal.

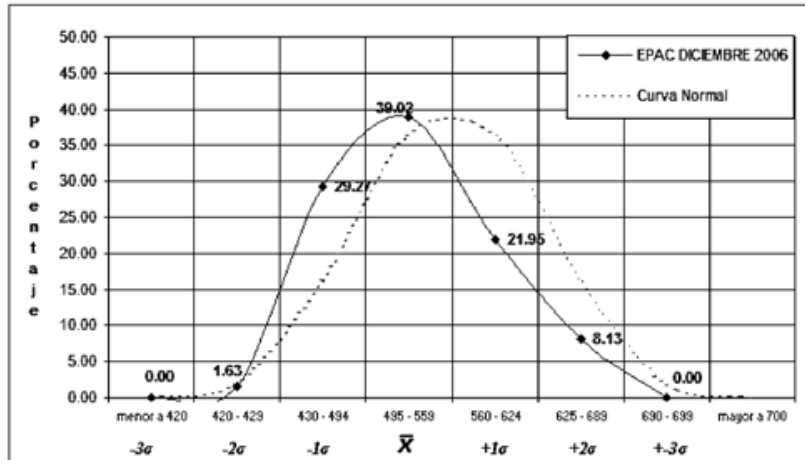

Bien sabemos que aún estamos en camino para alcanzar este panorama, no obstante, estamos seguros que a lo largo de dos años de trabajo constante se han colocado cimientos firmes que convierten al EPAC en un mecanismo sólido y confiable que contribuye a elevar la calidad de la formación de los profesionales de Enfermería.

\section{NOTAS DE REFERENCIA}

a Gaceta de la UNAM, 28 octubre de 2004.

b Se toma en cuenta para ser un buen reactivo el grado de dificultad (de 19.6 a 80.5) y el poder de discriminación (igual o mayor a 0.95).

c Aprobado por el H. Consejo Universitario el 11 de marzo de 1992. Actualización de los programas aprobada por el H. Consejo Técnico de la ENEO, Acuerdos 233/98 y 452/2000.

d Fuente: Planeación del EPAC, Unidad de Apoyo para la Evaluación de los Aprendizajes.

5,6 D. Stufflebeam, http://www.chasque.apc.org./gamolinar/ evaluacioneducativa.01.html\#anchor116783

7 Este artículo no incluye la última aplicación del EPAC que fue el 27 de octubre del 2007, con una participación de 1,688 sustentantes para el nivel licenciatura.

8 Fuente: resultados obtenidos de las Hojas de respuesta en los años 2005 y 2006.

9 www.eneo.unam.mx, entrando a la liga EPAC, en la que se solicita el número de cuenta del sustentante y el número de sede en que presentó el examen para desplegarse la página en que señala el resultado obtenido.

\section{REFERENCIAS BIBLIOGRÁFICAS:}

1 Tamayo y Tamayo, Mario (2006), El proceso de la investigación científica, $4^{\circ}$ edición, Ed. Noriega/Limusa, México.

2 Celman, Susana et al. (1998) La evaluación de los aprendizajes en el debate didáctico contemporáneo, Ed. Pardós, Buenos Aires.

3 Pérez Rocha, Manuel (1996) Evaluación, acreditación y calidad de la Educación Superior. En Materiales de apoyo a la evaluación educativa. CIEES, CU-NA-EVA, ANUIS y SEP, México.

4 Torres, Rosa María (2000) La Evaluación, Ediciones Novedades Educativas, Argentina.

\section{DIRECCIÓN DE CORRESPONDENCIA}

Mtra. Ma Antonia González Noriega: tgnoriega@mejico.com

Lic. Yazmin Nicolás Caballero: nulysus25@hotmail.com

Lic. Octavio Rodríguez Arellano: octavio@eneo.unam.mx 